\title{
Systematic analysis of therapeutic patterns and healthcare use during 12 months before inflammatory bowel disease-related hospitalization in Switzerland
}

\author{
Schoepfer, Alain ; Vavricka, Stephan R ; Brüngger, Beat ; Blozik, Eva ; Bähler, Caroline
}

\begin{abstract}
BACKGROUND AND AIMS Given the lack of data, we aimed to systematically analyze therapeutic patterns and health resource utilization in the year before inflammatory bowel disease (IBD)related hospitalization. METHODS Based on claims data of the Helsana health insurance group, therapy patterns and health resource utilization were assessed, and costs reimbursed by mandatory basic health insurance were calculated during a one-year period before an index hospitalization between 1 January 2013 to 31 December 2015. RESULTS We analyzed 344 IBD patients (140 ulcerative colitis [40.7\%], 204 Crohn's disease [59.3\%]). Drug regimens applied in the year before index hospitalization were as follows: no IBD drugs (43.6\% ulcerative colitis, 43.1\% Crohn's disease); 5-ASA (45.7\% ulcerative colitis, $19.1 \%$ Crohn's disease); local steroids (17.9\% ulcerative colitis, $17.6 \%$ Crohn's disease); systemic steroids (38.6\% ulcerative colitis, $29.4 \%$ Crohn's disease); immunomodulators (10.7\% ulcerative colitis, $18.1 \%$ Crohn's disease); biologics (10\% ulcerative colitis, $24 \%$ Crohn's disease); and calcineurin inhibitors $(2.1 \%$ ulcerative colitis, 1.5\% Crohn's disease). Forty-five percent of ulcerative colitis patients and $31.4 \%$ of Crohn's disease patients had no diagnostic procedures [computed tomography (CT), MRI, radiograph, sonography, colonoscopy, and calprotectin] in the year before hospitalization. Total annual health care costs before index hospitalization was EUR 4060 (interquartile range (IQR) 2360-7390) for ulcerative colitis and EUR 4900 (IQR 1520-14 880) for Crohn's disease patients, respectively. CONCLUSIONS Over $40 \%$ of ulcerative colitis and Crohn's disease patients did not receive any treatment in the year before index hospitalization. Efforts should be launched to timely diagnose and adequately treat IBD outpatients.
\end{abstract}

DOI: https://doi.org/10.1097/MEG.0000000000001616

Posted at the Zurich Open Repository and Archive, University of Zurich ZORA URL: https://doi.org/10.5167/uzh-181116

Journal Article

Published Version

Originally published at:

Schoepfer, Alain; Vavricka, Stephan R; Brüngger, Beat; Blozik, Eva; Bähler, Caroline (2020). Systematic analysis of therapeutic patterns and healthcare use during 12 months before inflammatory bowel diseaserelated hospitalization in Switzerland. European Journal of Gastroenterology Hepatology, 32(3):350-357. DOI: https://doi.org/10.1097/MEG.0000000000001616 


\title{
Systematic analysis of therapeutic patterns and healthcare use during 12 months before inflammatory bowel disease-related hospitalization in Switzerland
}

\author{
Alain Schoepfer ${ }^{a, b}$, Stephan R. Vavrickac ${ }^{c}$ Beat Brüngger ${ }^{d}$, Eva Blozik ${ }^{d}$ and Caroline Bähler ${ }^{d}$
}

Background and aims Given the lack of data, we aimed to systematically analyze therapeutic patterns and health resource utilization in the year before inflammatory bowel disease (IBD)-related hospitalization.

Methods Based on claims data of the Helsana health insurance group, therapy patterns and health resource utilization were assessed, and costs reimbursed by mandatory basic health insurance were calculated during a one-year period before an index hospitalization between 1 January 2013 to 31 December 2015.

Results We analyzed 344 IBD patients (140 ulcerative colitis [40.7\%], 204 Crohn's disease [59.3\%]). Drug regimens applied in the year before index hospitalization were as follows: no IBD drugs (43.6\% ulcerative colitis, $43.1 \%$ Crohn's disease); 5 -ASA (45.7\% ulcerative colitis, $19.1 \%$ Crohn's disease); local steroids (17.9\% ulcerative colitis, $17.6 \%$ Crohn's disease); systemic steroids (38.6\% ulcerative colitis, 29.4\% Crohn's disease); immunomodulators (10.7\% ulcerative colitis, 18.1\% Crohn's disease); biologics (10\% ulcerative colitis, $24 \%$ Crohn's disease); and calcineurin inhibitors (2.1\% ulcerative colitis, $1.5 \%$ Crohn's disease). Forty-five percent of ulcerative colitis patients and $31.4 \%$ of Crohn's disease patients had no diagnostic procedures [computed tomography (CT), MRI, radiograph, sonography, colonoscopy, and calprotectin] in the year before hospitalization. Total annual health care costs before index hospitalization was EUR 4060 (interquartile range (IQR) 23607390) for ulcerative colitis and EUR 4900 (IQR 1520-14 880) for Crohn's disease patients, respectively.

Conclusions Over $40 \%$ of ulcerative colitis and Crohn's disease patients did not receive any treatment in the year before index hospitalization. Efforts should be launched to timely diagnose and adequately treat IBD outpatients. Eur J Gastroenterol Hepatol XXX: 00-00

Copyright ( 2019 Wolters Kluwer Health, Inc. All rights reserved.

\section{Background}

Inflammatory bowel diseases (IBD) are chronic disabling disorders characterized by chronic inflammation of the gastrointestinal tract. Ulcerative colitis and Crohn's disease represent the two main forms of IBD. IBD seems to evolve in genetically susceptible individuals based on a dysbalanced gastrointestinal immune system that reacts inappropriately to microbes of the gastrointestinal tract [1]. Prevalence estimates range between 0.3 and $0.5 \%$, and are higher in Western industrialized countries $[2,3]$. Prevalence and incidence of IBD have increased in many, predominantly industrialized, countries [4]. IBD may not

\footnotetext{
European Journal of Gastroenterology \& Hepatology 2019, XXX:00-00 Keywords: healthcare costs, inflammatory bowel disease, inpatients

aDivision of Gastroenterology and Hepatology, Centre Hospitalier Universitaire Vaudois/CHUV, bUniversity of Lausanne (UNIL), Lausanne, Switzerland, 'Center for Gastroenterology and Hepatology, Vulkanplatz, Zurich-Altstetten and dDepartment of Health Sciences, Helsana Group, Zürich, Switzerland

Correspondence to Dr Caroline Bähler, PhD, Department of Health Sciences, Helsana Group, 8081 Zürich, Switzerland

Tel: +41 5834052 01; e-mail: caroline.baehler-baumgartner@helsana.ch

Received 5 August 2019 Accepted 23 October 2019

Supplemental Digital Content is available for this article. Direct URL citations appear in the printed text and are provided in the HTML and PDF versions of this article on the journal's website, www.eurojgh.com
}

only be limited to the gastrointestinal tract, patients may suffer also from associated comorbidities such as extraintestinal manifestations (arthralgias, skin manifestations such as erythema nodosum or psoriasis, etc.) $[5,6]$. These IBD-associated comorbidities may increase health resource utilization in terms of outpatient consultations, diagnostic procedures, therapeutic modalities (e.g., drug regimens), hospitalizations, and need for surgeries [7-9]. In a recently studied cohort of 4791 Swiss IBD patients, $78 \%$ had at least one comorbidity with a median of three chronic conditions [10]. The most prevalent chronic comorbidities were cardiovascular diseases $(41.4 \%)$, rheumatologic conditions $(36.3 \%)$, acid-related disorders $(26.5 \%)$, and pain $(25.8 \%)$ [10].

A recently published study from the Swiss IBD Cohort found that almost $14 \%$ of IBD patients were hospitalized for at least one day during a 12-month follow-up [11]. As of yet, no data is available regarding therapeutic strategies and healthcare usage in the 12 months before an IBDrelated hospitalization. The assessment of these factors is important in order to understand if suboptimal therapeutic strategies and monitoring of IBD patients contribute to the consecutive IBD-related hospitalization.

Healthcare claims data from insurance groups are very useful tools to evaluate prescribed drugs and health resource utilization for IBD patients in Switzerland. The 
claims data are reliable, practice-based and provide a high level of completeness that is independent of the insurance coverage. The Helsana Group covers a relatively large and geographically diverse part of the Swiss population. Our research group has recently published on the health resource utilization in IBD outpatients [12]. In outpatients, the discrimination between Crohn's disease and ulcerative colitis is not possible when analyzing health insurance claims data.

We conducted this study to evaluate the drug therapy patterns, health resource utilization, and the associated costs in the 12-month period preceding hospitalization.

\section{Methods}

\section{Study design}

This is a retrospective cohort study of patients with IBD who were hospitalized in the period from 1 January 2013 to 31 December 2015. Patients were followed during a 12-month period before being hospitalized due to an IBD flare (day 365 of the evaluation period corresponds to the day before the IBD-related hospitalization). Health resource utilization was assessed during the 12-month period before the hospitalization and costs were calculated for outpatient treatment, stratified into costs for primary care, specialist care, drugs (IBD related and non-IBD related), laboratory examinations, and others (paramedical visits, home care nursing services, and use of medical devices).

The study complies with Swiss federal legislation. All data were anonymized, retrospective, preexisting, and deidentified in order to protect the privacy of patients, physicians, and hospitals. Therefore, no ethics committee approval was necessary for this study.

\section{Study population}

The Helsana database underlying this study included mandatory health insurance claims from approximately 1.2 million persons per year, covering about $15 \%$ of the entire Swiss population (which counts currently about 8 million inhabitants). We identified 513 patients aged 18 years and older with at least one IBD-related hospitalization between 1 January 2013 and 31 December 2015.

Patients with a preceding hospitalization during the 12 months before the IBD-related index hospitalization $(n=139)$, deceased patients $(n=21)$, asylum seekers $(n=3)$, as well as individuals who dropped out of the Helsana Group during the 1 -year evaluation period $(n=6)$ were excluded in order to have the complete data for the given observation time. Of the 344 IBD patients included in the final sample, $140(57.2 \%)$ had ulcerative colitis and 204 patients $(42.8 \%)$ had Crohn's disease.

\section{Measures}

Since 2012, Switzerland has introduced a 'diagnosis-related group' system, which refers to ICD-10 codes and applied procedures. As such, IBD patients were identified using the ICD-10 codes K50 and K51, respectively. Further population characteristics were used as covariates. They included sex, age group (18-40, 41-60, 61+), additional chronic conditions, language area, type of insurance coverage (managed care model, supplementary hospital insurance, and deductible class), type of residence, as well as having had surgery at index hospitalization [13].

In Switzerland, health insurance is mandatory for each citizen and is based on a cost sharing compulsory basic coverage for each resident consisting of co-payments and deductibles. The height of the deductible can to some extent be chosen by the policy holder, whereby higher deductibles lead to lower monthly payments to the health insurance. Deductibles range from EUR 250 to 2060, whereby deductibles higher than EUR 410 belong to the higher deductible class for the purpose of this article. Co-payments amount to $10 \%$ of the yearly health care costs and are limited to EUR 580 per person per year.

\section{Drugs applied}

Following the concept of the therapeutic pyramid in IBD patients, we defined the following steps $[14,15]$ :

1. 5-aminosalicylic acid (mesalazine and sulfasalazine)

2. Locally active corticosteroids (budesonide)

3. Systemic corticosteroids (prednisone)

4. Immunomodulators (thiopurines, methotrexate)

5. Biologics (Tumor necrosis factor alpha (TNF) antagonists: adalimumab, infliximab, golimumab, certolizumab-pegol; integrin inhibitors: vedolizumab)

6. Others (calcineurin inhibitors: tacrolimus).

\section{Healthcare costs}

We aimed to determine the economic burden of hospitalized IBD patients from a social insurance payer perspective including all type of direct health care costs (i.e., taxfunded costs are excluded). Annual total healthcare costs in IBD patients in the year before index hospitalization in the time period from 1 January 2013 to 31 December 2015 were obtained from providers' claims and defined as the total amount of outpatient costs per patient per 12-month period with mandatory insurance coverage. Costs were not limited to an IBD indication. As such, costs for IBDassociated comorbidities such as rheumatologic manifestations are included in the analysis. Costs from the outpatient setting comprised payments for office-based physician visits (primary care physician and specialists), hospital outpatient visits, paramedical visits (e.g., physiotherapists), home care nursing services, medications, diagnostic procedures (laboratory tests, imaging), and medical devices.

All costs are converted and quoted in Euros (1 $\mathrm{CHF}=0.824$ EUR; effective 30 June 2014). Outpatient costs are covered by the health insurance, inpatient costs to a share between 45 and $55 \%$ (depending on the canton and calendar year). Out-of-pocket payments, depending on the chosen premium, could not be considered, because they are not covered by the compulsory health insurance.

\section{Healthcare utilization}

Outpatient visits were not limited to an IBD indication. Visits were defined as direct contacts between patient and physician (including home visits). As such, visits for treatment with TNF antagonists were also considered to be medical visits if a face-to-face contact with a physician took place. The following supplementary measures are 
provided as outpatient visits in the 1-year observation period: the total number of outpatient visits, the number of primary care physician and specialist visits (stratified into gastroenterologists, including hospital outpatient visits, and other specialists), as well as the proportion of patients without any gastroenterologist visit in the look-back period. We performed a detailed analysis of IBD-related drugs used in the observation interval. Furthermore, we evaluated the frequency and costs of tools for diagnosis and monitoring of IBD, such as abdominal computed tomography (CT) scanning, abdominal and/or pelvic MRI examinations, radiograph examinations, abdominal ultrasounds, endoscopies, and fecal calprotectin.

\section{Statistical analysis}

Bivariate descriptive statistics were used to show differences between the characteristics of individuals with either Crohn's disease or ulcerative colitis using Fisher's exact test for dichotomous variables, Wilcoxon rank sum test for continuous, and Chi-squared test for categorical variables. Direct healthcare costs and healthcare utilization are presented overall and then stratified according to Crohn's disease or ulcerative colitis diagnosis.

Treatment with IBD-specific drugs in the year before hospitalization was analyzed using transversal state distribution plots and stacked bar charts, both following the concept of the therapeutic pyramid in IBD (see chapter methods, drugs applied) [15,16]. Furthermore, we used line plots of smoothed data (where the smoothing method was local polynomial regression fitting) to show temporal changes in drug prescriptions. All analyses were carried out using R, version 3.2.0 (R Core Team. R: A Language and Environment for Statistical Computing. 2015. http:// www.R-project.org/) [17].

\section{Results}

\section{Patient characteristics and drugs used in the year before hospitalization}

The baseline characteristics of the 344 patients are shown in Table 1. When compared with ulcerative colitis patients, Crohn's disease patients were younger (median 45 vs 61 years, $P<0.001)$, they had less comorbid conditions
$(P=0.003)$, and they underwent significantly more frequently an IBD-related surgical intervention at index hospitalization $(26.0$ vs $7.1 \%, P<0.001)$.

The drugs used during the 1-year period prior to hospitalization are shown in Table 2. A total of $43.3 \%$ of patients did not have any IBD-related drugs, with no difference being observed between ulcerative colitis and Crohn's disease patients. When compared with ulcerative colitis patients, Crohn's disease patients were significantly less frequently treated with 5 -ASA ( 19.1 vs $45.7 \%$, $P<0.001$ ), and they received more frequently anti-TNF drugs $(24.0$ vs $9.3 \%, P<0.001)$. Following the concept of the therapeutic pyramid, we evaluated which 'highest' drug category was applied in the year prior to hospitalization (Supplementary Table 1, Supplemental digital content 1, http://links.lww.com/EJGH/A484). We observed a significant overall difference between Crohn's disease and ulcerative colitis patients. When compared with ulcerative colitis patients, Crohn's disease patients more frequently received biologic therapies. The proportion of ulcerative colitis and Crohn's disease patients who were treated with an IBD-related drug, following again the principle of the therapeutic pyramid, is shown in Fig. 1. Figure 1 demonstrates the large proportion of Crohn's disease and ulcerative colitis patients without any IBD-specific drug during the year before the hospitalization. 5-ASA only was used much more frequently in ulcerative colitis patients compared with Crohn's disease patients. Steroids as the 'highest' drug category, in combination with 5-ASA, were found more frequently in ulcerative colitis compared with Crohn's disease patients. Compared with ulcerative colitis patients, Crohn's disease patients more often had a step-up to biologic therapies. Figure 2 provides a detailed analysis of the prescription pattern of the distinct IBD-specific drugs during the year before hospitalization, stratified according to IBD diagnosis. A mild increase in 5-ASA prescriptions was observed in the last 3 months before hospitalization in Crohn's disease patients, while this increase was marked in ulcerative colitis patients. A similar pattern was observed for the prescription of steroids, again with a mild increase for Crohn's disease patients in the months before hospitalization which was contrasted by a marked increase for ulcerative colitis patients. The prescription of immunomodulators was relatively constant from month 12 to 4

Table 1. Baseline characteristics of included patients $(n=344)$

\begin{tabular}{|c|c|c|c|c|}
\hline & Total IBD & Ulcerative colitis patients & Crohn's disease patients & $P$ value \\
\hline$n$ & 344 & $140(40.7 \%)$ & $204(59.3 \%)$ & \\
\hline Female sex & $185(53.8 \%)$ & $81(57.9 \%)$ & $104(51.0 \%)$ & ns \\
\hline Age (years, median, first-third quartile) & $49(33,69)$ & $61(36,74)$ & $45(31,62)$ & $<0.001$ \\
\hline Age groups (years) & & & & $<0.001$ \\
\hline $18-40$ & $127(36.9 \%)$ & $42(30.0 \%)$ & $85(41.7 \%)$ & \\
\hline $41-60$ & $92(26.7 \%)$ & $27(19.3 \%)$ & $65(31.9 \%)$ & \\
\hline $60+$ & $125(36.3 \%)$ & $71(50.7 \%)$ & $54(26.5 \%)$ & \\
\hline Type of residence (urban) & $269(78.2 \%)$ & $106(75.7 \%)$ & $163(79.9 \%)$ & ns \\
\hline French/Italian (vs German) region & $76(22.1 \%)$ & $30(21.4 \%)$ & $46(22.5 \%)$ & ns \\
\hline Chronic conditions [mean, median (first-third quartile)] & $2.4,2.0(1.0-4.0)$ & $2.8,2.0(1.0-4.0)$ & $2.1,2.0(1.0-3.0)$ & 0.003 \\
\hline \multicolumn{5}{|l|}{ Health insurance plan } \\
\hline Managed care & $159(46.2 \%)$ & $74(52.9 \%)$ & $85(41.7 \%)$ & 0.047 \\
\hline Higher deductible & $43(12.5 \%)$ & $13(9.3 \%)$ & $30(14.7 \%)$ & ns \\
\hline Supplementary hospital insurance & $58(16.9 \%)$ & $25(17.9 \%)$ & $33(16.2 \%)$ & ns \\
\hline Surgery at index hospitalization & $63(18.3 \%)$ & $10(7.1 \%)$ & $53(26.0 \%)$ & $<0.001$ \\
\hline
\end{tabular}

$P$ values, assigning the differences between ulcerative colitis and Crohn's disease, were calculated using fisher's exact test for dichotomous variables, Wilcoxon rank sum test for continuous, and Chi-squared test for categorical variables.

IBD, inflammatory bowel disease; ns, not significant. 
Table 2. Drugs used by inflammatory bowel disease patients during the one-year follow period prior to the inflammatory bowel disease-related hospitalization

\begin{tabular}{|c|c|c|c|c|}
\hline & Total IBD & Ulcerative colitis patients & Crohn's disease patients & $P$ value \\
\hline$n$ & 344 & $140(40.7 \%)$ & 204 (59.3\%) & \\
\hline No IBD-related drugs & 149 (43.3\%) & $61(43.6 \%)$ & $88(43.1 \%)$ & ns \\
\hline $5-A S A$ & $103(29.9 \%)$ & $64(45.7 \%)$ & $39(19.1 \%)$ & $<0.001$ \\
\hline Steroids (total) & $143(41.6 \%)$ & $65(46.4 \%)$ & $78(38.2 \%)$ & ns \\
\hline Systemic & $114(33.1 \%)$ & 54 (38.6\%) & 60 (29.4\%) & ns \\
\hline Local & $61(17.7 \%)$ & 25 (17.9\%) & 36 (17.6\%) & ns \\
\hline Immunmodulators & $52(15.1 \%)$ & $15(10.7 \%)$ & $37(18.1 \%)$ & ns \\
\hline TNF antagonists (TNF) & 62 (18.0\%) & $13(9.3 \%)$ & 49 (24.0\%) & $<0.001$ \\
\hline Integrin inhibitors & $1(0.3 \%)$ & $1(0.7 \%)$ & $0(0 \%)$ & na \\
\hline Biologics (TNF + INT) & 63 (18.3\%) & $14(10.0 \%)$ & 49 (24.0\%) & 0.001 \\
\hline Calcineurin inhibitors & $6(1.7 \%)$ & $3(2.1 \%)$ & $3(1.5 \%)$ & na \\
\hline
\end{tabular}

$P$ values, assigning the differences between ulcerative colitis and Crohn's disease, were calculated using fisher's exact test.

IBD, inflammatory bowel disease; INT, integrin inhibitors; na, not applicable related to small sample size; ns, not significant; TNF, tumor necrosis factor alpha.
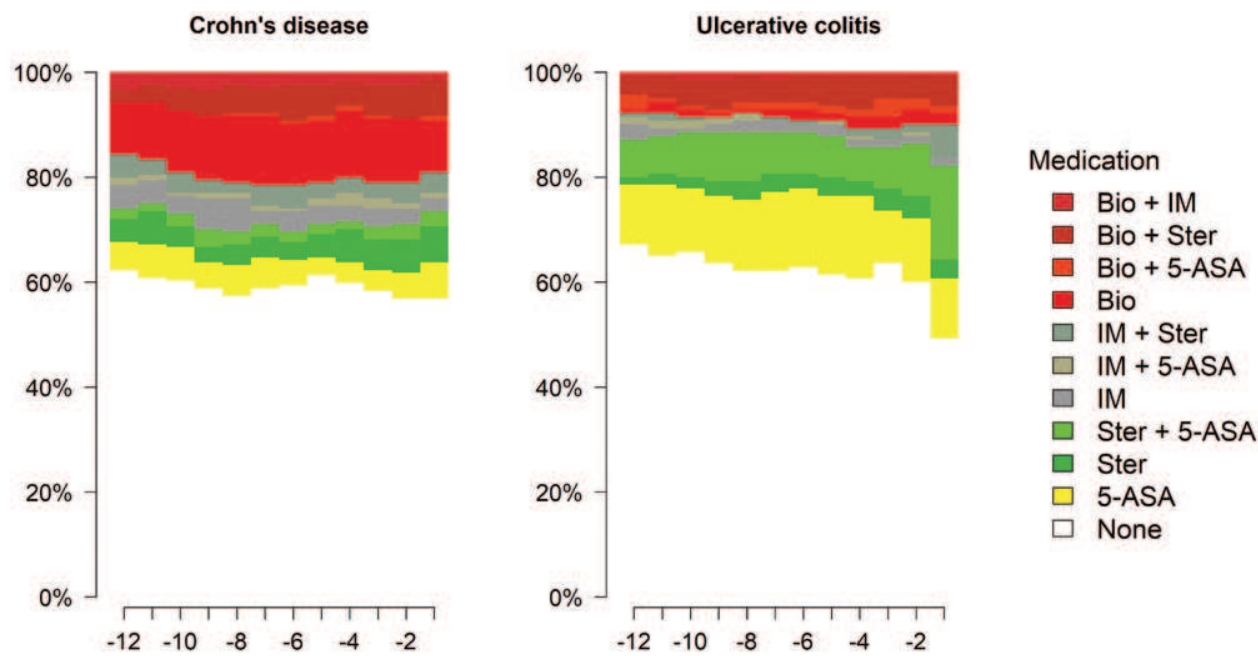

Fig. 1. Proportion of ulcerative colitis and Crohn's disease patients with an IBD-related drug in the year before IBD-related hospitalization. 5-ASA, aminosalicylates; Bio, biologics; IBD, inflammatory bowel disease; IM, immunomodulators; Ster, steroids.

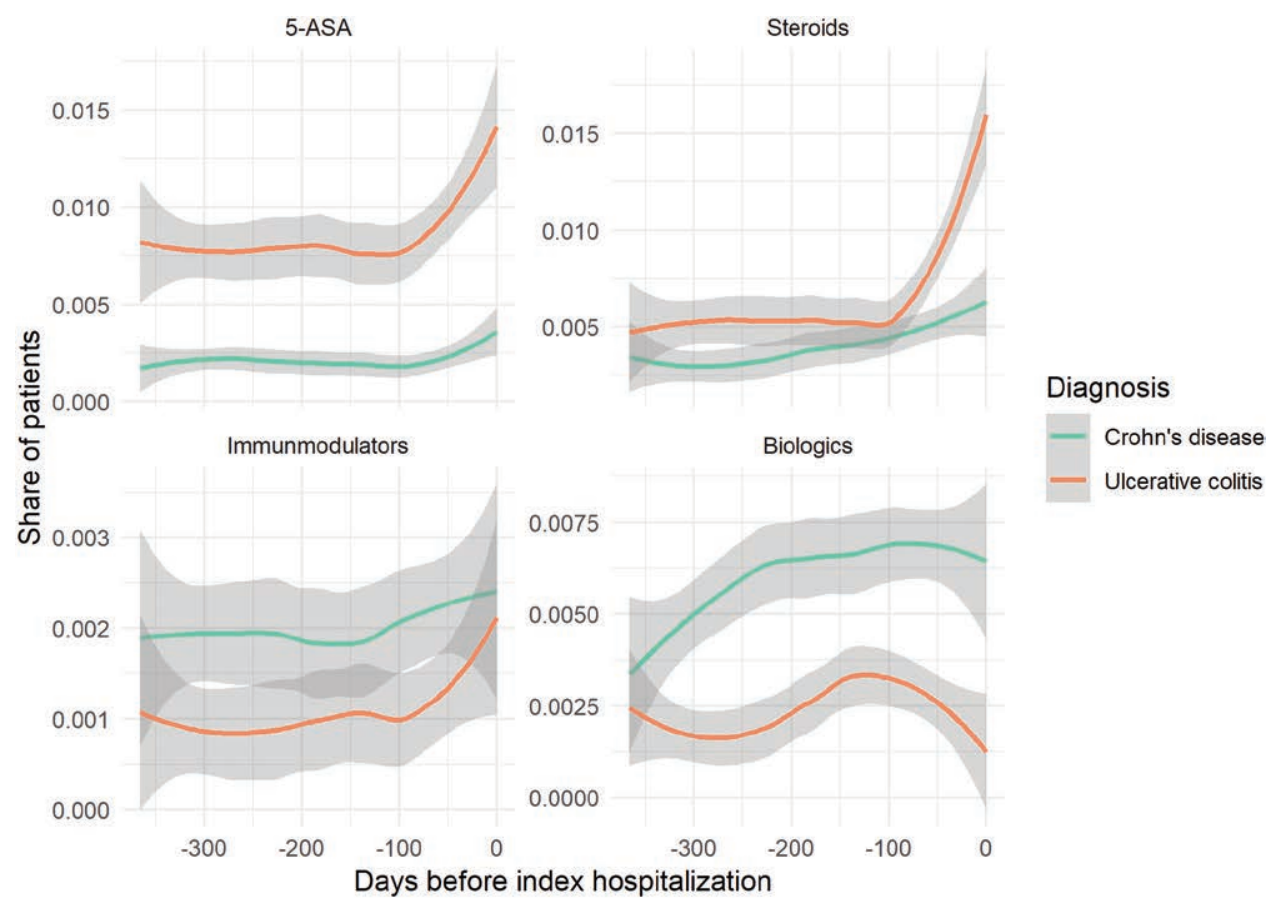

Fig. 2. Proportion of ulcerative colitis and Crohn's disease patients with an IBD-related drug in the 12-month period before hospitalization. IBD, inflammatory bowel disease. 
before hospitalization, whereas it gradually increased for both ulcerative colitis and Crohn's disease in the 3 months before hospitalization. Biologic use was characterized by a gradual increase with a plateau at 3 months before hospitalization in Crohn's disease patients compared with a peak in ulcerative colitis patients at month 4 before hospitalization, but then a steep decrease in the last 3 months before hospitalization.

Compared with patients not undergoing surgery at index hospitalization, IBD patients with surgery were significantly more often treated with local steroids (25.4 vs $16.0 \%, P=0.047$ ), immunomodulators (33.3 vs $11.0 \%$, $P<0.001)$, and biological therapy $(39.7$ vs $13.5 \%$, $P<0.001)$ in the 12 months prior to hospitalization.

The healthcare plan (managed care, basic insurance, and supplementary insurance) had no influence on the frequency of prescribed IBD drugs in the 12 months prior to hospitalization, with the expection of 5-ASA in Crohn's disease patients which were found significantly less frequently in patients with managed care plan compared with patients with basic health insurance (11.8 vs $24.4 \%, P=0.030)$.

We further analyzed the drug prescription patterns stratified according to the observation if patients had at least one visit with a gastroenterologist in the last 3 months before the hospitalization. Supplementary Fig. 1, Supplemental digital content 2, http://links.lww.com/ EJGH/A485 shows indeed that the $52.9 \%$ of ulcerative colitis patients who had at least one visit with a gastroenterologist within the last 3 months before the hospitalization were characterized by a much higher frequency of prescription of IBD-specific drugs when compared with ulcerative colitis patients who did not have a visit with a gastroenterologist within the last 3 months before the hospitalization. A marked increase in the prescription of 5-ASA, steroids, and immunomodulators in the last 3 months before hospitalization could be observed for patients who consulted a gastroenterologist, while biologic treatment was more frequent in these patients throughout the 1-year observation period.

Supplementary Fig. 2, Supplemental digital content 3, http://links.lww.com/EJGH/A486 shows the IBD-specific drug prescriptions in the year before hospitalization in the $59.8 \%$ of Crohn's disease patients who had at least one visit with a gastroenterologist within the last 3 months before the hospitalization compared with the group without a visit with a gastroenterologist. Crohn's disease patients with at least one visit to the gastroenterologist in the last 3 months prior to hospitalization were prescribed significantly more frequently steroids, immunomodulators, and biologics when compared to their counterparts, with no significant difference regarding 5-ASA prescriptions. Similar to the observation in ulcerative colitis patients, a gradual increase in the prescription of IBD-specific drugs was observed over time.

\section{Diagnostic procedures in the year before hospitalization}

The frequencies of abdominal CT scanning, abdominal and/or pelvic MRI examinations, radiograph examinations, abdominal ultrasounds, colonoscopies, as well as calprotectin measurements in the 12-months look-back period are shown in Table 3. A total of $36.9 \%$ of IBD patients had no diagnostic procedures at all in the year before hospitalization. Diagnostic procedures (endoscopy/sonography and/or fecal calprotectin) were significantly more often performed in Crohn's disease patients compared with ulcerative colitis patients $(65.7$ vs $50.7 \%$, $P=0.007)$. We further evaluated if there is a difference in diagnostic procedures with regards to therapy with IBDspecific drugs. When comparing ulcerative colitis patients who received IBD drug treatment in the 12 months before hospitalization with ulcerative colitis patients without IBD drug treatment, we found that ulcerative colitis patients under IBD drugs underwent significantly more often diagnostic procedures $(75.9$ vs $27.9 \%, P<0.001$, Supplementary Table 2, Supplemental digital content 1, http://links.lww.com/EJGH/A484). The same was found for Crohn's disease patients where $86.2 \%$ of patients under IBD drugs underwent diagnostic procedures compared with $45.5 \%$ of patients without IBD drug treatment $(P<0.001$, Supplementary Table 3, Supplemental digital content 1, http://links.lww.com/EJGH/A484).

We further evaluated the concordance between the patients without any diagnostic procedures and patients who did not receive any IBD-specific treatment in order to assess if both populations are identical. Of the $36.9 \%$ of patients without any diagnostic procedures, $72.4 \%$ did not receive any IBD-specific drugs. On the other hand, out of the $43.3 \%$ of patients without IBD-specific drugs, a total of $61.7 \%$ did not undergo a diagnostic procedure. As such we can conclude that both groups have a relevant overlap but they are not identical (Cramer's V of 0.450 which is regarded as moderate association).

\section{Consultations in ulcerative colitis and Crohn's disease patients}

We further analyzed the number of face-to-face consultations in the one-year period before hospitalization (Table 4). The median number of face-to-face

Table 3. Diagnostic procedures in ulcerative colitis and Crohn's disease patients in the year before hospitalization

\begin{tabular}{|c|c|c|c|c|}
\hline & Total IBD & ulcerative colitis patients & Crohn's disease patients & $P$ value \\
\hline$n$ & 344 & $140(40.7 \%)$ & $204(59.3 \%)$ & \\
\hline No diagnostic procedures & $127(36.9 \%)$ & $63(45.0 \%)$ & $64(31.4 \%)$ & 0.012 \\
\hline CT & $45(13.1 \%)$ & $12(8.6 \%)$ & $33(16.2 \%)$ & ns \\
\hline MRI & $44(12.8 \%)$ & $5(3.6 \%)$ & $39(19.1 \%)$ & $<0.001$ \\
\hline Radiograph & $8(2.3 \%)$ & $3(2.1 \%)$ & $5(2.5 \%)$ & na \\
\hline Sonography & $126(36.6 \%)$ & $40(28.6 \%)$ & $86(42.2 \%)$ & 0.012 \\
\hline Colonoscopy & $121(35.2 \%)$ & $43(30.7 \%)$ & $78(38.2 \%)$ & ns \\
\hline Calprotectin & $91(26.5 \%)$ & $24(17.1 \%)$ & $67(32.8 \%)$ & 0.001 \\
\hline Endoscopy/Sonography/Calprotectin & $205(59.6 \%)$ & $71(50.7 \%)$ & $134(65.7 \%)$ & 0.007 \\
\hline
\end{tabular}

$P$ values, assigning the differences between ulcerative colitis and Crohn's disease, were calculated using fisher's exact test.

$\mathrm{CT}$, computed tomography; IBD, inflammatory bowel disease; na, not applicable; ns, not significant. 


\begin{tabular}{|c|c|c|c|c|}
\hline Consultations (median, first-third quartile) & Total IBD & ulcerative colitis patients & Crohn's disease patients & $P$ value \\
\hline$n$ & 344 & $140(40.7 \%)$ & $204(59.3 \%)$ & \\
\hline Face-to-face consultations & $13.5(7.0-22.0)$ & $15.0(7.0-22.5)$ & $12.5(7.0-22.0)$ & ns \\
\hline By primary care physicians & $5.0(1.0-10.0)$ & $6.0(3.0-11.0)$ & $4.0(1.0-9.0)$ & 0.002 \\
\hline By gastroenterologists & $3.0(0-9.0)$ & $2.5(0-7.0)$ & $3.0(0-10.0)$ & ns \\
\hline By other specialists & $1.0(0-5.0)$ & $1.0(0-5.0)$ & $1.0(0-4.25)$ & ns \\
\hline Patients (\%) without any visit by gastroenterologists in look-back & $96(27.9 \%)$ & $41(29.3 \%)$ & $55(27.0 \%)$ & ns \\
\hline
\end{tabular}

$P$ values, assigning the differences between ulcerative colitis and Crohn's disease, were calculated using Wilcoxon rank sum test IBD, inflammatory bowel disease; na, not applicable; ns, not significant.

\begin{tabular}{|c|c|c|c|c|}
\hline Costs (median, first-third quartile) & Total IBD & ulcerative colitis patients & Crohn's disease patients & $P$ value \\
\hline Outpatient & $4320(1910-11890)$ & $4060(2360-7390)$ & $4900(1520-14880)$ & ns \\
\hline Primary care & $360(110-690)$ & $430(190-790)$ & $290(30-610)$ & 0.003 \\
\hline Specialists & 1370 (330-2650) & $1220(320-2300)$ & $1600(350-2780)$ & ns \\
\hline Medications & $1300(400-4080)$ & $1280(580-2920)$ & $1310(310-8880)$ & ns \\
\hline Laboratory & $390(150-850)$ & $380(130-770)$ & $410(150-930)$ & ns \\
\hline Others & $50(0-370)$ & $70(0-540)$ & $40(0-270)$ & ns \\
\hline
\end{tabular}

$P$ values, assigning the differences between ulcerative colitis and Crohn's disease, were calculated using Wilcoxon rank sum test; other costs include costs incurred by paramedical visits (e.g., physiotherapists), home care nursing services and by use of medical devices.

ns, not significant

consultations was 13.5 (interquartile range (IQR) 7-22), of which a median of 5 (IQR 1-10) consultations were provided by primary care physicians and a median of 3 (IQR 0-9) consultations by gastroenterologists. In bivariate analyses, the number of consultations was not different between Crohn's disease and ulcerative colitis patients, except for visits to primary care physicians. We further evaluated the number of consultations stratified according to IBD diagnosis and according to the fact if patients were treated with IBD-specific drugs or not. When compared with ulcerative colitis patients without IBD drugs, ulcerative colitis patients under IBD drugs significantly more frequently had consultations by any physician (median 16 vs $11, P=0.002$ ), and consultations by a gastroenterologist (median 5 vs. $1, P<0.001$, Supplementary Table 4, Supplemental digital content 1, http://links.lww.com/ EJGH/A484). Similar results were observed in Crohn's disease patients (Supplementary Table 5, Supplemental digital content 1, http://links.lww.com/EJGH/A484), where patients under IBD drugs significantly more frequently had consultations by any physician (median 17 vs. $8, P<0.001)$, and consultations by a gastroenterologist (median 8 vs. $1, P<0.001$ ).

\section{Costs in ulcerative colitis and Crohn's disease patients in the year prior to hospitalization}

We further evaluated the total costs of healthcare utilization during the year prior to hospitalization (Table 5). In the unadjusted bivariate analysis, the median annual outpatient costs per IBD patient were EUR 4320 with almost equal costs for Crohn's disease and ulcerative colitis patients. Costs for specialist consultations were higher compared with the costs for primary care. Drug costs accounted for roughly $30 \%$ of the outpatient costs without any difference between ulcerative colitis and Crohn's disease patients. Interestingly, the majority of drug costs was associated with non-IBD-related drugs whereas IBDrelated drug costs accounted for less than $10 \%$ of the median drug costs.

\section{Discussion}

This is the first population-based in-depth study that examines the drug prescription patterns, health resource utilization, and costs reimbursed by basic health insurance during a 12-month period before an IBD-related index hospitalization. Our analysis contains several clinically relevant messages. First, $43 \%$ of IBD patients were not treated with IBD-specific drugs in the observation period and $37 \%$ of these patients did not undergo any diagnostic procedures. Second, patients being followed by gastroenterologists had significantly more frequent treatment with IBD-specific drugs when compared with patients followed by primary care physicians. Third, prescription of IBDspecific drugs increased in frequency during the three months before IBD-related hospitalization. We observed relevant differences between ulcerative colitis and Crohn's disease patients regarding the drug prescription patterns with immunomodulators and biologic therapy being more frequently used in Crohn's disease patients compared with ulcerative colitis patients. And last, median outpatient costs in the 12-month observation period were similar in both diseases with about EUR 4300 .

The first key finding is that roughly $40 \%$ of IBD patients were neither treated with IBD-specific drugs nor did they undergo any diagnostic or follow-up procedure in the 12 months before IBD-related hospitalization. Several reasons might explain this finding. The first reason might be that patients were diagnosed with IBD only during hospitalization. In fact, diagnostic delay has been identified as a common problem in IBD. In Switzerland, median diagnostic delay was determined to be 9 months for Crohn's disease patients compared with 4 months for patients with ulcerative colitis [18]. Timely diagnosis of IBD is relevant not only to decrease suffering on patient's side but also to decrease the risk of bowel damage that is associated with increased risk of intestinal surgery in Crohn's disease patients [19]. The Swiss findings have been corroborated by several other studies $[20,21]$. The broader use of fecal calprotectin among healthcare practitioners might have 
the potential to reduce diagnostic delay in IBD patients [22]. Another reason for not receiving IBD-specific treatment could be IBD patients could have been asymptomatic in the year before IBD-related hospitalization and treating physicians might have seen no necessity for treatment and/or diagnostic procedures. Furthermore, patients might have been nonadherent to the prescription of IBDspecific drugs. In fact, nonadherence has been identified as a relevant problem in IBD. A threshold of following treatment advice for at least $80 \%$ of the time has been accepted as standard for good adherence and was applied in most studies [23]. Nonadherence strongly depends on drug classes used and vary from 7 to $72 \%$ with an overall nonadherence rate around roughly $35 \%$. The $43 \%$ of IBD patients not receiving any IBD-specific treatment in our cohort are close to the reported overall nonadherence rate. Our database did not allow to answer the question how many patients were newly diagnosed with IBD during the hospitalization. As for the $37 \%$ of IBD patients not having had any diagnostic and/or follow-up procedures in the year before IBD-related hospitalization, this number compares well with the $43 \%$ of patients not being treated with IBD-specific drugs. It is crucial to remember that IBD patients need regular follow-up visits even if they are asymptomatic as also patients in clinical remission might have ongoing endoscopically active disease that is associated with bowel damage in the long term [22,24-26]. We observed that IBD patients who were followed by gastroenterologists received more frequently IBD drug treatment (5-ASA, steroids, immunomodulators, and biologics) when compared with patients who were followed by primary care physicians. Apparently, there exists a difference in treatment patterns between gastroenterologists and primary care physicians in the 12-month period before IBDrelated hospitalization. However, our data do not allow to judge if this observation is related to differences in disease activity.

IBDs are known to be associated with important health resource utilization and associated costs $[27,28]$. In a systematic review comparing the costs of Crohn's disease in the United States with costs in Western industrialized countries, researchers found that estimated direct annual costs were US\$ 18 022-18932 for Crohn's disease patients in the United States and EUR 2898-6960 in other Western countries [29]. Of note, the differences in healthcare systems between various countries somehow limits direct comparisons. Median costs for healthcare consumption in the year before IBD-related hospitalization was EUR 4320 with comparable costs for Crohn's disease and ulcerative colitis patients. IBD drugs and specialist consultations each accounted for one third of total costs. As such, these costs are much lower compared with the costs that were generated during an IBD-related hospitalization and an observation period of one year (starting with the day of the index hospitalization) where total median costs were approximately EUR 14230 for ulcerative colitis patients and EUR 20000 for Crohn's disease patients [30]. In hospitalized IBD patients, about one-third of total costs was attributed to inpatient costs, whereas IBD drugs were the highest cost position for outpatients [29]. Several studies reported on a shift from inpatient to outpatient costs in recent years, which seems closely related to the introduction of new medical therapies such as biologics, which are used more frequently in Crohn's disease than in ulcerative colitis patients $[7,13]$.

Our study has several strengths and also some limitations. The first strength is that our analysis relies on a large study population which enabled us to provide an overview of the IBD situation in Switzerland. This is in contrast to data from the Swiss IBD cohort study where patients are included in $80 \%$ by gastroenterologists working in hospital. As such, our sample can be regarded as population-based. We think that our data are representative of the situation in Switzerland, as Helsana covers $15 \%$ of the entire Swiss population. Second, our data are innovative as this is the first study to provide a detailed analysis of the use of IBD-specific drugs and healthcare utilization in the 12 months before IBD-related hospitalization. Third, data were not collected by means of self-report and, as such, results were not skewed due to an inherent recall bias. A fourth strength is that claims data in hospitalized IBD patients allow us to stratify into ulcerative colitis and Crohn's disease which stands in contrast to Swiss claims data from the outpatient setting. Based on this, we were able to reveal significant differences between the two entities with regards to the utilization of IBDspecific drugs but also healthcare utilization. As a first limitation, data did not allow us to answer the question of how many patients were newly diagnosed with IBD when they were hospitalized. As such, we cannot judge the proportion of newly diagnosed IBD patients was among those who did not receive any IBD-specific drug during the 12-month period before hospitalization. Second, we have no information on disease duration and disease course. Both factors could have a direct impact on drug use, healthcare utilization, and costs. However, following legal obligations, health insurances are not allowed to capture these data.

In conclusion, nearly half of IBD patients did not receive any IBD-specific treatment and close to $40 \%$ had no diagnostic procedures in the 12 months before hospitalization. The frequency of use of 5-ASA, steroids, and immunomodulators typically increased in the three months before hospitalization. Median annual costs before IBD-related hospitalization were about EUR 4300. Our data imply that a relevant proportion of IBD patients is not benefiting from adequate diagnostic and therapeutic measures. With the ultimate goal of improving quality of care and associated health-related outcomes, measures should be taken to timely diagnose and treat IBD outpatients and monitor their adherence to treatment.

\section{Acknowledgements}

This study was funded by MSD Merck Sharp and Dohme AG (Switzerland). The sponsor reviewed, discussed and approved the protocol CH-LCE-6247/2019-MS-1296. Conduction, analysis and interpretation of the data, preparation and submission of the manuscript were solely in the responsibility of the authors, without any obligations to the sponsor.

A.S., S.V., B.B., E.B., and C.B. conceptualized and designed the study. C.B. and BB performed the statistical analysis. All authors contributed to data interpretation and manuscript writing and had full access to all data in the study. All authors read and approved the final manuscript. 
The manuscript, including related data, figures and tables has not been previously published and that the manuscript is not under consideration elsewhere

According to the Swiss Federal Law on data protection, this study was exempted from ethics committee approval as all data were anonymized, retrospective, preexisting, and deidentified in order to protect the privacy of patients, physicians, and hospitals.

Individual data cannot be made fully available because the study is based on claims data of the Helsana Group, the owner of the data. Thus, data underlie data protection and privacy restrictions. These restrictions prohibit us from sharing the collected data.

\section{Conflicts of interest}

A.S. consulted and received speaker's honoraria from Abbvie, UCB, Falk, MSD, Tillotts, Vifor, Janssen, Pfizer, Ferring, Receptos, and Takeda. S.R.V. consulted and received speaker's honoraria from Abbvie, UCB, Falk, Janssen, MSD, Tillots, Vifor, Pfizer, Takeda, and Ferring. There are no conflicts of interest for the remaining authors.

\section{References}

1 Ananthakrishnan AN. Epidemiology and risk factors for IBD. Nat Rev Gastroenterol Hepatol 2015; 12:205-217.

2 Kappelman MD, Rifas-Shiman SL, Kleinman K, Ollendorf D, Bousvaros A, Grand RJ, Finkelstein JA. The prevalence and geographic distribution of Crohn's disease and ulcerative colitis in the United States. Clin Gastroenterol Hepatol 2007; 5:1424-1429.

3 Manninen P, Karvonen AL, Huhtala H, Rasmussen M, Collin P. The epidemiology of inflammatory bowel diseases in Finland. Scand $J$ Gastroenterol 2010; 45:1063-1067.

4 Molodecky NA, Soon IS, Rabi DM, Ghali WA, Ferris M, Chernoff G, et al. Increasing incidence and prevalence of the inflammatory bowel diseases with time, based on systematic review. Gastroenterology 2012; 142:46-54.e42; quiz e30.

5 Vavricka SR, Brun L, Ballabeni P, Pittet V, Prinz Vavricka BM, Zeitz J, et al. Frequency and risk factors for extraintestinal manifestations in the Swiss inflammatory bowel disease cohort. Am J Gastroenterol 2011; 106:110-119.

6 Vavricka SR, Schoepfer A, Scharl M, Lakatos PL, Navarini A, Rogler G. Extraintestinal manifestations of inflammatory bowel disease. Inflamm Bowel Dis 2015; 21:1982-1992.

7 van der Valk ME, Mangen MJ, Leenders M, Dijkstra G, van Bodegraven $\mathrm{AA}$, Fidder $\mathrm{HH}$, et al.; COIN study group and the Dutch Initiative on Crohn and Colitis. Healthcare costs of inflammatory bowel disease have shifted from hospitalisation and surgery towards anti-TNF $\alpha$ therapy: results from the COIN study. Gut 2014; 63:72-79.

8 Burisch J, Jess T, Martinato M, Lakatos PL; ECCO -EpiCom. The burden of inflammatory bowel disease in Europe. J Crohns Colitis 2013; 7:322-337.

9 Kappelman MD, Rifas-Shiman SL, Porter CQ, Ollendorf DA, Sandler RS, Galanko JA, Finkelstein JA. Direct health care costs of Crohn's disease and ulcerative colitis in US children and adults. Gastroenterology 2008; 135:1907-1913.

10 Bähler C, Schoepfer AM, Vavricka SR, Brüngger B, Reich O. Chronic comorbidities associated with inflammatory bowel disease: prevalence and impact on healthcare costs in Switzerland. Eur J Gastroenterol Hepatol 2017; 29:916-925.

11 Sulz MC, Siebert U, Arvandi M, Gothe RM, Wurm J, von Känel R, et al.; Swiss IBD Cohort Study Group. Predictors for hospitalization and outpatient visits in patients with inflammatory bowel disease: results from the Swiss inflammatory bowel disease cohort study. Eur J Gastroenterol Hepatol 2013; 25:790-797.

12 Bähler C, Vavricka SR, Schoepfer AM, Brüngger B, Reich O. Trends in prevalence, mortality, health care utilization and health care costs of swiss IBD patients: a claims data based study of the years 2010, 2012 and 2014. BMC Gastroenterol 2017; 17:138.

13 Huber CA, Szucs TD, Rapold R, Reich O. Identifying patients with chronic conditions using pharmacy data in Switzerland: an updated mapping approach to the classification of medications. BMC Public Health 2013; 13:1030

14 Safroneeva E, Vavricka SR, Fournier N, Straumann A, Rogler G, Schoepfer AM. Prevalence and risk factors for therapy escalation in ulcerative colitis in the swiss IBD cohort study. Inflamm Bowel Dis 2015; 21:1348-1358.

15 Rogler G. Where are we heading to in pharmacological IBD therapy? Pharmacol Res 2015; 100:220-227.

16 Gabadinho A, Ritschard G, Müller NS, Studer M. Analyzing and visualizing state sequences in R with TraMineR. J Stat Softw 2011; 40:1-37.

17 R Core Team. R: A Language and Environment for Statistical Computing. 2015. http://www.R-project.org/.

18 Vavricka SR, Spigaglia SM, Rogler G, Pittet V, Michetti P, Felley C, et al.; Swiss IBD Cohort Study Group. Systematic evaluation of risk factors for diagnostic delay in inflammatory bowel disease. Inflamm Bowel Dis 2012; 18:496-505.

19 Schoepfer AM, Dehlavi MA, Fournier N, Safroneeva E, Straumann A, Pittet V, et al.; IBD Cohort Study Group. Diagnostic delay in Crohn's disease is associated with a complicated disease course and increased operation rate. Am J Gastroenterol 2013; 108:1744-1753; quiz 1754.

20 Novacek G, Gröchenig HP, Haas T, Wenzl H, Steiner P, Koch R, et al. Diagnostic delay in patients with inflammatory bowel disease in Austria. Wien Klin Wochenschr 2019; 131:104-112.

21 Lee DW, Koo JS, Choe JW, Suh SJ, Kim SY, Hyun JJ, et al. Diagnostic delay in inflammatory bowel disease increases the risk of intestinal surgery. World J Gastroenterol 2017; 23:6474-6481.

22 Mumolo MG, Bertani L, Ceccarelli L, Laino G, Di Fluri G, Albano E, et al. From bench to bedside: fecal calprotectin in inflammatory bowel diseases clinical setting. World J Gastroenterol 2018; 24:3681-3694.

23 Lenti MV, Selinger CP. Medication non-adherence in adult patients affected by inflammatory bowel disease: a critical review and update of the determining factors, consequences and possible interventions. Expert Rev Gastroenterol Hepatol 2017; 11:215-226.

24 Naganuma M, Hisamatsu T, Matsuoka K, Kiyohara H, Arai M, Sugimoto $\mathrm{S}$, et al. Endoscopic severity predicts long-term prognosis in Crohn's disease patients with clinical remission. Digestion 2016; 93:66-71.

25 Falvey JD, Hoskin T, Meijer B, Ashcroft A, Walmsley R, Day AS, Gearry RB. Disease activity assessment in IBD: clinical indices and biomarkers fail to predict endoscopic remission. Inflamm Bowel Dis 2015; 21:824-831.

26 Schoepfer AM, Beglinger C, Straumann A, Trummler M, Vavricka SR, Bruegger LE, Seibold F. Fecal calprotectin correlates more closely with the simple endoscopic score for Crohn's disease (SES-CD) than CRP, blood leukocytes, and the CDAl. Am J Gastroenterol 2010; 105:162-169.

27 Park KT, Bass D. Inflammatory bowel disease-attributable costs and cost-effective strategies in the United States: a review. Inflamm Bowel Dis 2011; 17:1603-1609.

28 Gibson TB, Ng E, Ozminkowski RJ, Wang S, Burton WN, Goetzel RZ, Maclean R. The direct and indirect cost burden of Crohn's disease and ulcerative colitis. J Occup Environ Med 2008; 50:1261-1272.

29 Yu AP, Cabanilla LA, Wu EQ, Mulani PM, Chao J. The costs of Crohn's disease in the United States and other western countries: a systematic review. Curr Med Res Opin 2008; 24:319-328.

30 Schoepfer A, Vavricka SR, Brüngger B, Reich O, Blozik E, Bähler C. Systematic analysis of annual health resource utilization and costs in hospitalized patients with inflammatory bowel disease in Switzerland. Eur J Gastroenterol Hepatol 2018; 30:868-875. 